\title{
Assistência de enfermagem a gestantes usuárias de crack e cocaína: revisão integrativa
}

\author{
Nursing care for pregnant crack and cocaine users: an integrative review
}

Asistencia de enfermería a embarazadas usuarias de crack y cocaína: revisión integradora

\begin{abstract}
Caroline Valério Clementino' ๑; Gabriella de Andrade Boskall ๑; Júlia Carolina de Mattos Cerioni Silva' ๑;

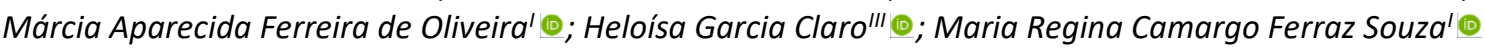

'Universidade de São Paulo, São Paulo, SP, Brasil; "Universidade Estadual do Centro-Oeste, Guarapuava, PR, Brasil; "'Universidade Estadual de Campinas, Campinas, SP, Brasil

\begin{abstract}
RESUMO
Objetivo: identificar na literatura evidências relacionadas à assistência de enfermagem a gestantes usuárias de crack e cocaína nos serviços de saúde. Método: revisão integrativa realizada em julho de 2020, com publicações dos últimos dez anos nas bases de dados Embase (Medline e PubMed), PsycINFO, Scopus e Cinahl. Resultados: foram encontrados 49 artigos, sendo incluídos dois internacionais e quatro nacionais. Dentre os conceitos que fundamentam a assistência de enfermagem às gestantes usuárias de crack e cocaína destacam-se: acolhimento, vínculo e encaminhamentos a serviços especializados. Três estudos abordam o despreparo e desconhecimento do enfermeiro diante deste cuidado e constata-se a infrequência de atitudes consonantes com a política de saúde mental. Conclusão: o acompanhamento integral e sistematizado pelo enfermeiro durante o período gestacional é indispensável e necessita de aprimoramento na linha da redução de danos. Os achados sugerem estratégias diferenciadas para a assistência de enfermagem e reforçam as lacunas práticas e científicas relacionadas ao tema. Descritores: Enfermagem; Gestação; Cocaína Crack; Cocaína; Redução de Dano.
\end{abstract}

\begin{abstract}
Objective: to identify, in the literature, evidence relating to nursing care for pregnant women crack and cocaine users in health services. Method: this integrative review was conducted in July 2020, with publications from the previous ten years in the Embase (Medline and PubMed), PsycINFO, Scopus, and Cinahl databases. Results: the search strategy found 49 research papers and included two international and four national studies. Among the concepts that underlie nursing care for pregnant women who use crack and cocaine, the following stand out: supportive reception, bonding, and referral to specialized services. Three studies addressed nurses' lack of preparedness and knowledge in providing such care, and attitudes aligned with mental health policy were found to be infrequent. Conclusion: systematic, comprehensive monitoring by nurses is essential during the gestational period and the harm reduction component needs improvement. The findings recommend differentiated nursing care strategies and underline the gaps in the practice and science relating to this issue.

Descriptors: Nursing; Pregnancy; Crack Cocaine; Cocaine, Harm Reduction.
\end{abstract}

\section{RESUMEN}

Objetivo: identificar en la literatura evidencias relacionadas con la atención de enfermería a embarazadas que consumen crack y cocaína en los servicios de salud. Método: revisión integradora realizada en julio de 2020, con publicaciones de los últimos diez años en las bases de datos Embase (Medline y PubMed), PsycINFO, Scopus y Cinahl. Resultados: se encontraron 49 artículos, siendo dos internacionales y cuatro nacionales. Entre los conceptos que sustentan la atención de enfermería a las embarazadas usuarias crack y cocaína, destacan: acogida, vinculación y derivación a servicios especializados. Tres estudios abordan la falta de preparación y el desconocimiento de los enfermeros en cuanto a esta atención. Se constata también la falta de actitudes alineadas con la política de salud mental. Conclusión: el seguimiento integral y sistemático por parte de enfermeros durante el período gestacional es fundamental y necesita perfeccionamiento en la reducción de daños. Los hallazgos sugieren estrategias de asistencia de enfermería diferenciadas y refuerzan las brechas prácticas y científicas relacionadas con el tema. Descriptores: Enfermería; Embarazo; Cocaina Crack; Cocaina; Reducción del Daño.

\section{INTRODUÇÃO}

Estima-se que dentre as 29 milhões de pessoas com problemas relacionados ao uso de álcool e outras drogas em todo o mundo, 18,3 milhões seja associado a cocaína e seus derivados, sobretudo o crack, com um aumento significativo do público feminino nestes perfis epidemiológicos ${ }^{1}$.

No Brasil, em 2017, a cocaína foi usada por 0,9\% e o crack por 0,3\% da população². A pesquisa nacional identificou aproximadamente 370 mil usuários de crack no país e deste total, 78,8 mil (21,3\%) são mulheres. Além disso, as mulheres representam $20 \%$ dos usuários frequentadores de cenas de uso de crack e vivenciam uma realidade bastante vulnerável, considerando sua baixa escolaridade, violências sofridas, exposição a riscos para fazer o uso, uso concomitante com substâncias lícitas, dentre outras questões ${ }^{3,4}$. 
Aproximadamente $46 \%$ das brasileiras que consomem crack relataram quatro ou mais gestações ao longo da vida. Das que gestaram ao menos uma vez, 64,6\% referiram pelo menos uma gravidez após ter iniciado o uso de crack e/ou similares, ademais, $49 \%$ apresentaram ao menos uma gestação que não evoluiu ao nascimento ou que resultou em feto natimorto ${ }^{3}$. Alguns determinantes para o uso de substâncias pelas gestantes relacionam-se a gravidez indesejada, a continuidade do uso para evitar abstinência, a influência do parceiro do gênero masculino, como também, o duplo efeito da gravidez como facilitador ou moderador deste uso, fatores estes frequentemente identificados em mulheres que vivem em condições de vulnerabilidade ${ }^{5,6}$.

Com relação aos cuidados em saúde durante o período gestacional, apenas 52,5\% das usuárias referiram ter procurado atendimentos em Unidades Básicas de Saúde (UBS), Centros de Saúde, Ambulatórios, Unidades de Pronto Atendimento (UPAs). Ou seja, metade das gestantes usuárias de crack não acessam qualquer serviço de saúde, chamando a atenção para o baixo acompanhamento pré-natal ${ }^{3}$. Outro problema é a falta de abordagem pelos próprios profissionais de saúde sobre a questão do consumo de drogas na gestação e estes, quando abordam, são de forma superficial com orientações insuficientes ${ }^{7}$.

O trabalho do enfermeiro é considerado constituinte na assistência a gestantes de alto risco. Este geralmente faz o primeiro contato com essas mulheres na atenção primária e na atenção secundária realiza a consulta completa e acompanhamento de todo o pré-natal junto da equipe multiprofissional com identificação dos problemas e potenciais da gestante e elaboração do plano de ação singular para cada mulher, sendo corresponsável pelas metas pactuadas entre todos os envolvidos ${ }^{8,9}$

Para isso, os profissionais de enfermagem necessitam de competências técnicas mínimas de abordagem psicossocial e assim contribuir na prevenção de agravos e desfechos indesejáveis na gestação e parto ${ }^{10}$. A Organização Mundial da Saúde (OMS) recomenda cinco princípios básicos para o atendimento de gestantes usuárias de substâncias psicoativas: 1- priorizar a prevenção; 2- garantir o acesso aos serviços de saúde; 3- respeitar a autonomia da usuária; 4prestar uma assistência de cuidado integral; 5- proteger contra qualquer discriminação e estigmatização ${ }^{11}$.

Diante deste cenário, este estudo tem por objetivo identificar na literatura as principais evidências científicas relacionadas à assistência de enfermagem a gestantes usuárias de crack e cocaína nos serviços de saúde, que possam amparar a prática profissional.

\section{MÉTODO}

Estudo descritivo, com delineamento de revisão integrativa da literatura (RI) que consiste em método amplo, com a finalidade de sintetizar as pesquisas sobre um determinado assunto, permitindo a inclusão de estudos de diferentes abordagens metodológicas. A RI visa obter uma conclusão, que passa a constituir fonte de conhecimento sobre o problema avaliado para ser transferido para a prática clínica dos profissionais nos serviços de saúde, no ensino, na gestão e na formulação de políticas públicas, com base nos resultados de pesquisas que investigaram problemas idênticos ou similares ${ }^{12}$.

A RI foi conduzida por dois revisores independentes e organizada em cinco fases: 1) identificação do problema e elaboração da questão de pesquisa; 2) pesquisa bibliográfica com estratégia de pesquisa abrangente; 3) avaliação dos dados pela representatividade dos estudos primários encontrados; 4) análise de dados com síntese, apresentação, comparações e conclusões; e 5) apresentação final dos resultados com a descrição das implicações práticas, políticas e para a pesquisa e as limitações da revisão ${ }^{13}$.

A fase 1 se deu pelas inquietações e problemas vivenciados no cuidado a gestantes usuárias de drogas associado a escassez de orientações científicas para a atuação do enfermeiro. A estratégia PICo, acrônimo de $P$ (População) $=$ Gestantes usuárias de crack e cocaína; I (Intervenção) = Assistência de Enfermagem e Co (Contexto) = Serviços de Saúde ${ }^{14}$, foi utilizada para a elaboração da questão norteadora do estudo, que consistiu em: Qual o conhecimento disponível na literatura sobre a assistência de enfermagem prestada nos serviços de saúde a gestantes usuárias de crack e cocaína?

A estratégia de busca e seleção dos estudos foram realizadas por um pesquisador e uma bibliotecária, de forma independente, em julho de 2020, em quatro bases de dados indexadas: Embase (Medline e o PubMed), PsycINFO, Scopus e CINAHL, utilizando a combinação dos Descritores em Ciências da Saúde (DECS), da Biblioteca Virtual em Saúde e da Medical Subject Headings (MeSH) com a seguinte estratégia para todas as bases de dados de forma abrangente: (Pregnant Woman) OR (Pregnancy) OR (Expectant Mother) AND (Cocaine, Crack) OR (Cocaine Related Disorder) OR (Cocaine Dependence) OR (Cocaine Addiction) OR (crack abuse) OR (crack dependence) AND (nursing) OR (nursing care) OR (nursing assistance).

Na fase 3, foram definidos os seguintes critérios de inclusão: estudos primários na íntegra, publicados nos últimos 10 anos, até a data limite de 30 de junho de 2020 (data da coleta de dados), nos idiomas português, inglês e espanhol, em revistas indexadas, que abordassem a assistência de enfermagem a gestantes usuárias de crack e cocaína; Foram excluídos estudos da literatura cinzenta que não representavam a temática do estudo, capítulos de livro, resumos de estudos apresentados em Congressos, dissertações de mestrado, teses de doutorado e relatórios técnicos. 
Após a identificação dos artigos, estes foram exportados para o software gerenciador de referências Mendeley, buscando identificar os artigos duplicados e reunir todas as publicações encontradas. A seleção dos estudos seguiu as recomendações do método Perferred Reporting Items for Systematic Reviews and Meta-Analyses (PRISMA) ${ }^{15}$, representada na Figura 1.

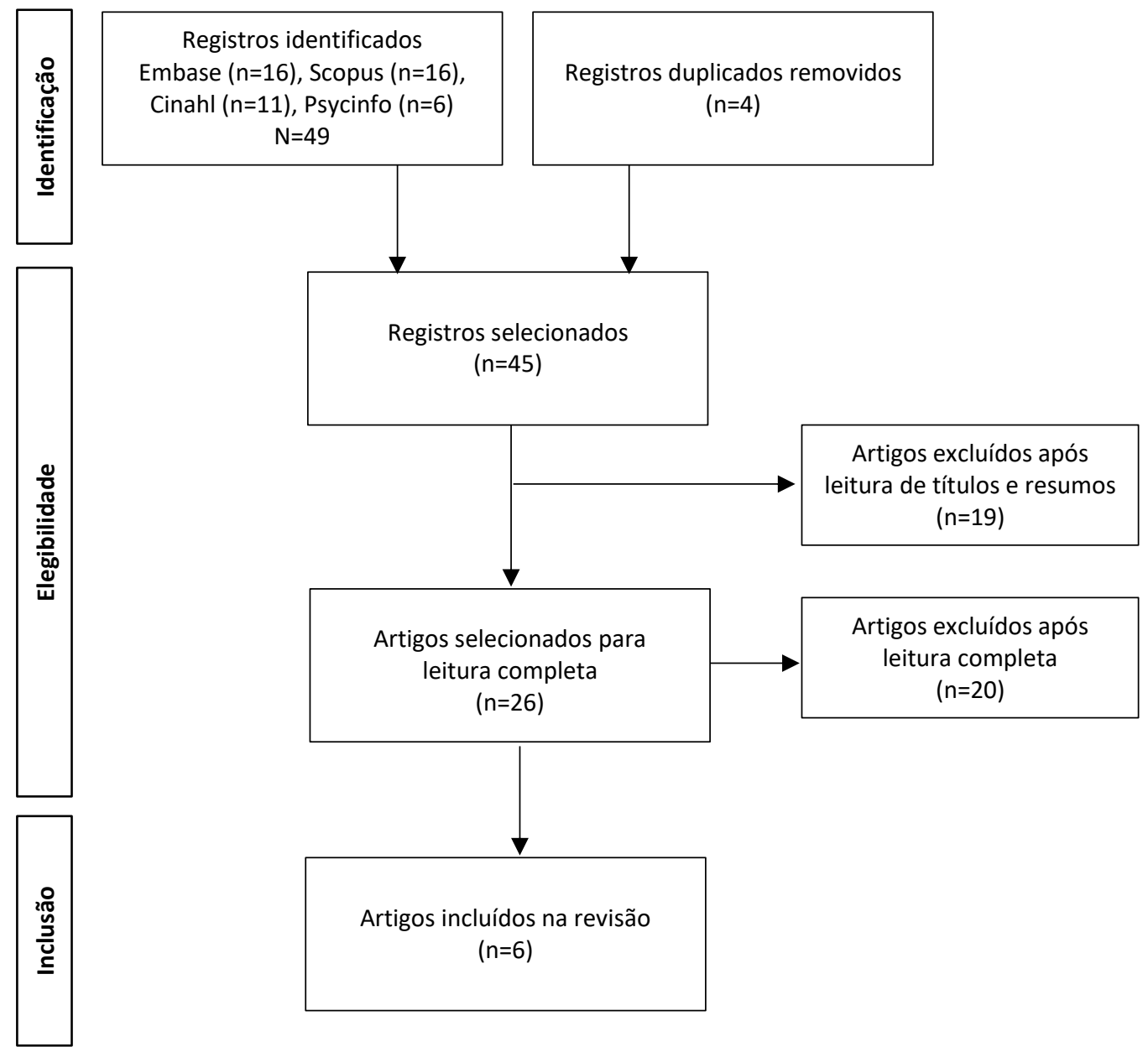

FIGURA 1: Fluxograma de acordo com o Perferred Reporting Items for Systematic Reviews and Meta-Analyses $\left(\right.$ PRISMA) ${ }^{16}$. São Paulo, SP, Brasil, 2020

$\mathrm{Na}$ fase 4, os dados foram analisados e organizados por meio de um instrumento construído pelos pesquisadores com base nos seguintes tópicos: título do artigo (identificação), ano de publicação, país de publicação (local de realização), características metodológicas, contexto do estudo, resultados e conclusões. A fase 5 é apresentada na seção de resultados deste estudo.

\section{RESULTADOS}

Um total de 49 estudos atenderam aos critérios de inclusão. Quatro foram identificados como duplicatas (artigo registrado simultaneamente em mais de uma base de dados), 39 foram excluídos e seis artigos compuseram a amostra final de artigos analisados nesta revisão.

Dentre os excluídos, 16 (41\%) abordavam a síndrome alcoólica fetal, 14 (35,9\%) o uso de opióides e outras substâncias durante a gestação, cinco apenas dados epidemiológico e/ou as consequências do consumo (12,8\%) e quatro resultaram de literatura cinzenta $(10,3 \%)$.

Os seis artigos foram publicados entre 2010 e 2020, destacando-se o ano de 2015 com duas destas publicações. Os contextos de realização das pesquisas foram a atenção especializada/hospitalar $(n=3)$ e a atenção primária ( $n=3$ ).

Entre os periódicos destacou-se a Escola Anna Nery Revista de Enfermagem com dois (33\%) estudos publicados. Em relação ao país de publicação, 71,4\% (4) são do Brasil, seguido de Austrália 14,3\% (1) e Canadá 14,3\% (1). Quanto 
ao método adotado nos estudos selecionados para a revisão todos os $100 \%$ (6) são descritivos, quatro são de abordagem qualitativa (71,4\%), um é de abordagem quantitativa (14,3\%) e um $(14,3 \%)$ é etnográfico.

Os principais conceitos que fundamentam a assistência de enfermagem mencionados pelos estudos da revisão foram: acolhimento, estabelecimento de vínculo entre profissional e gestante e encaminhamentos para os outros serviços de saúde.

Na Figura 2, apresenta-se a caracterização dos estudos incluídos na revisão.

\begin{tabular}{|c|c|c|c|c|}
\hline пo & Título/ Ano/País & $\begin{array}{c}\text { Delineamento/ } \\
\text { Contexto }\end{array}$ & Resultados & Conclusões \\
\hline 1 & $\begin{array}{l}\text { O enfermeiro frente ao } \\
\text { pré-natal das gestantes } \\
\text { usuárias de } \text { urack }^{17} \text {. } \\
2020 \\
\text { Brasil }\end{array}$ & $\begin{array}{l}\text { Estudo descritivo e } \\
\text { exploratório, de } \\
\text { abordagem qualitativa } \\
\text { realizado em Unidades } \\
\text { de Saúde da Família. }\end{array}$ & $\begin{array}{l}\text {-Enfermeiros não possuem capacitação para a } \\
\text { abordagem ao pré-natal de gestantes usuárias de } \\
\text { crack; } \\
\text { - Realizam o pré-natal conforme o manual do } \\
\text { Ministério da Saúde (procedimentos); } \\
\text { - Realizam a busca ativa einterconsulta com o } \\
\text { Núcleos de Apoio a Saúde da Família (NASF); } \\
\text { - Buscam acolher, aproximar, aconselhar e } \\
\text { estabelecer vínculo com a gestante. }\end{array}$ & $\begin{array}{l}\text { A diminição ou até mesmo a } \\
\text { suspensão do uso de } \\
\text { cocaína/crack pelas gestantes foi } \\
\text { maior naquelas que tiveram } \\
\text { maior número de consultas pré- } \\
\text { natal. }\end{array}$ \\
\hline 2 & $\begin{array}{l}\text { O papel do enfermeiro } \\
\text { durante a consulta de pré- } \\
\text { natal à gestante usuária de } \\
\text { drogas }^{18} \text {. } \\
2015 \\
\text { Brasil }\end{array}$ & $\begin{array}{l}\text { Estudo descritivo, } \\
\text { exploratório de caráter } \\
\text { quantitativo realizado } \\
\text { emuma UBS. }\end{array}$ & $\begin{array}{l}\text { - Realizam acolhimento, mas não questionam } \\
\text { sobre o uso de drogas, limitam-se a perguntar se } \\
\text { fazem uso ou não; } \\
\text { - Não utilizam a estratégia da Política de Redução } \\
\text { de Danos; } \\
\text { - Encaminhamentos não são qualificados; } \\
\text { - Baixa frequência de Visitas Domiciliares. }\end{array}$ & $\begin{array}{l}\text { Apesar dos enfermeiros } \\
\text { possuirem conhecimentos sobre } \\
\text { o uso de drogas e suas } \\
\text { consequências na gestação; eles } \\
\text { não dispõem de mecanismos } \\
\text { concretos para agir diante dessas } \\
\text { situações. }\end{array}$ \\
\hline 3 & $\begin{array}{l}\text { Self-care theory in } \\
\text { pregnant women during } \\
\text { chemical detoxification } \\
\text { from crack: } \text { nursing's } \\
\text { contributions }{ }^{19} \text {. } \\
2015 \\
\text { Brasil }\end{array}$ & $\begin{array}{l}\text { Estudo descritivo, } \\
\text { exploratório de } \\
\text { abordagem qualitativa, } \\
\text { realizado emuma } \\
\text { unidade de internação } \\
\text { para desintoxicação de } \\
\text { crack e outras drogas } \\
\text { de um hospital geral de } \\
\text { médio porte. } \\
\end{array}$ & $\begin{array}{l}\text { - Acolhem desde o início do pré-natal; } \\
\text { - Estimulam a reorganização da rotina e a prática } \\
\text { do autocuidado; } \\
\text {-Proporcionam ambiente favorável para o } \\
\text { cuidado; } \\
\text { - Estimulam a desintoxicação }\end{array}$ & $\begin{array}{l}\text { As atividades de auto cuidado } \\
\text { (propostas por Dorothea Orem) } \\
\text { possibilitou um cuidado de forma } \\
\text { ampliada, explorando o potencial } \\
\text { de cada gestante, evidenciando } \\
\text { suas qualidades. }\end{array}$ \\
\hline 4 & $\begin{array}{l}\text { Percepções e práticas de } \\
\text { gestantes atendidas na } \\
\text { atenção primária frente ao } \\
\text { uso de drogas }{ }^{20} \text {. } \\
2014 \\
\text { Brasil }\end{array}$ & $\begin{array}{l}\text { Estudo descritivo, } \\
\text { exploratório de } \\
\text { abordagem qualitativa } \\
\text { realizado em } 25 \text { UBS. }\end{array}$ & $\begin{array}{l}\text { - Julgam o consumo de drogas durante o pré- } \\
\text { natal; } \\
\text { - Não abordam ou abordam de forma insuficiente } \\
\text { a questão das drogas durante o pré-natal; } \\
\text { - Encaminham a gestante para outros serviços; } \\
\text { - Não ofereceram apoio suficiente às gestantes } \\
\text { (falta de continuidade no atendimento). }\end{array}$ & $\begin{array}{l}\text { Os profissionais precisam estar } \\
\text { sensibilizados e preprarados para } \\
\text { a abordagem das gestantes } \\
\text { usuárias de crack/cocaína, para } \\
\text { isso é preciso investimentos em } \\
\text { pesquisas que subsidiem essa } \\
\text { prática. }\end{array}$ \\
\hline 5 & $\begin{array}{l}\text { Participant perception of } \\
\text { an integrated program for } \\
\text { substance abuse in } \\
\text { pregnancy }{ }^{21} \text {. } \\
2010 \\
\text { Canadá }\end{array}$ & $\begin{array}{l}\text { Estudo descritivo de } \\
\text { abordagem qualitativa, } \\
\text { realizado em uma } \\
\text { Clínica Escola Médica e } \\
\text { em um Centro de } \\
\text { Prática de um Hospital } \\
\text { Geral. }\end{array}$ & $\begin{array}{l}\text { - Propiciam ambiente que facilite o retorno dessa } \\
\text { gestante ao Pré - Natal; } \\
\text { - Abordagem sem julgamento (manutenção de } \\
\text { atitudes positivas); } \\
\text { - Encaminhamento para outros serviços. }\end{array}$ & $\begin{array}{l}\text { A falta de cooperação das } \\
\text { gestantes usuárias de } \\
\text { crack/cocaína provoca a } \\
\text { conformidade nos profissionais } \\
\text { no que se refere à mudança de } \\
\text { atitude. Para o sucesso da } \\
\text { assistência faz-se necessária o } \\
\text { envolvimento de uma equipe } \\
\text { multidisciplinar em saúde mental } \\
\text { com acompanhamento para } \\
\text { além do período pós natal. }\end{array}$ \\
\hline 6 & $\begin{array}{l}\text { Drugs and having babies: } \\
\text { An exploration of how a } \\
\text { specialist clinic meets the } \\
\text { needs of chemically } \\
\text { dependent pregnant } \\
\text { women }^{22} \text {. } \\
2012 \\
\text { Austrália }\end{array}$ & $\begin{array}{l}\text { Estudo etnográfico } \\
\text { crítico realizado em } \\
\text { uma clínica } \\
\text { especializada para } \\
\text { usuárias de drogas. }\end{array}$ & $\begin{array}{l}\text { - O cuidado pode ser oferecido individual ou em } \\
\text { grupo; } \\
\text { - Realiza Aconselhamento; } \\
\text { - Trabalha na perspectiva da Redução de danos; } \\
\text { - Visita domiciliares à gestante ou a seus } \\
\text { familiares. }\end{array}$ & $\begin{array}{l}\text { Não se aplica, pois trata-se de } \\
\text { Protocolo Clínico }\end{array}$ \\
\hline
\end{tabular}

FIGURA 2: Caracterização dos estudos primários incluídos na revisão. São Paulo,SP, Brasil, 2020. 


\section{DISCUSSÃo}

Os estudos analisados apresentaram escassez de evidências sobre a assistência de enfermagem às gestantes usuárias de crack e cocaína, visto que foram encontrados apenas seis estudos relacionados à temática, dos quais quatro foram realizados no Brasil. A revisão ainda aponta dificuldades do enfermeiro frente ao cuidado bem como a falta de alinhamento às diretrizes da Organização Mundial da Saúde (OMS) e da Política de Saúde Mental. A revisão apontou maior número de publicações no ano de $2015^{16,17}$, o que indica interesse recente, ainda que os estudos sejam escassos, pela temática.

O preconceito é apontado dentre as inúmeras barreiras que as mulheres usuárias de substâncias ilícitas encontram ao acessar serviços de saúde ${ }^{16-18}$.

Estudo desenvolvido no Brasil ${ }^{19}$, com 25 mulheres usuárias de drogas em uma Unidade Básica de Saúde, do estado do Paraná, constatou que o preconceito se multiplica durante a gestação e, por constrangimento, tendem a não relatar o uso de drogas. Isso estende-se a não procurar ajuda ou procurá-la apenas em casos de urgência e, muitas dão entrada nos serviços de saúde somente no momento do parto, o que intensifica agravos e dificulta o trabalho preventivo.

Dificuldades em conseguir encaminhamentos para serviço especializado e a continuidade do atendimento no período gestacional, também foi relatado pelas mulheres. Destaca-se também que a maior parte das informações recebidas pela gestante têm centralidade no bebê e em muitos casos restritas a dizer que as drogas prejudicam, bem como a recomendação da suspensão do uso, afastando as mesmas dos cuidados e até mesmo culpabilizando-as ${ }^{19}$.

Quanto à assistência de enfermagem, o estudo realizado em Alagoas, em Unidade Básica de Saúde, com 50 gestantes usuárias de álcool e outras drogas, apresenta que $100 \%$ delas não foram abordadas pelo enfermeiro para participarem do pré-natal, $70 \%$ quando foram atendidas por outras razões, receberam cuidado precário, em $86 \%$ o enfermeiro apenas perguntou se elas consumiam algum tipo de substância psicoativa ou não, 92\% não foram orientadas sobre nenhum cuidado relacionado à redução de danos e nenhuma delas foi orientada sobre tratamento e/ou encaminhamento ao Centro de Atenção Psicossocial Álcool e Drogas (CAPSad), se necessário ${ }^{17}$.

O consumo de substâncias ilícitas durante a gestação possui repercussões e julgamentos sociais ainda maiores. Consonante a este acontecimento, parte fundamental do trabalho do enfermeiro consiste em preparar a equipe de saúde para manejar este fenômeno na comunidade, objetivando a promoção da assistência à saúde e a redução de riscos e danos ${ }^{20}$. Alguns profissionais utilizam da educação em saúde associada às consultas de pré-natal e às visitas domiciliares visando a redução dos agravos, para promover o esclarecimento popular sobre o uso de crack e cocaína durante a gestação, objetivando a diminuição dos estigmas sociais e o acesso ao cuidado em saúde ${ }^{21}$.

$\mathrm{O}$ acolhimento e o estabelecimento de vínculo são apontados em quatro estudos como conceitos predominantes na assistência de enfermagem ${ }^{16-18,20}$.

O acolhimento e o vínculo são ferramentas essenciais para garantir a qualidade de qualquer atendimento contínuo em saúde e um dos recursos do enfermeiro nesta construção é a relação terapêutica entre enfermeiro e gestante, que promove a escuta qualificada, diálogo, empatia, apoio, aceitação e confiança ${ }^{16}$. Por meio desta relação, totalmente isenta de julgamentos, as orientações serão recebidas pela mulher e impactarão no acesso aos cuidados de enfermagem de forma qualificada ${ }^{20}$.

A criação de ambiente terapêutico no atendimento da gestante foi apontada por dois estudos, como de extrema importância e que pode ser desenvolvida por meio do atendimento individual ou em grupo ${ }^{18,21}$, pois muitas mulheres referem o desejo de participarem de atividades nos espaços de saúde junto com as outras gestantes para compartilhar seus anseios com relação à maternidade e não se sentirem excluídas pelo seu consumo de álcool ou outras drogas ${ }^{20}$.

No entanto, os seis estudos incluídos nesta revisão apontam evidências em relação ao uso de substâncias psicoativas na gestação como uma dificuldade da assistência de enfermagem, pois são casos que suscitam demandas pessoais, morais e éticas dos profissionais, sobretudo quando a gestante não escolhe, ou não consegue, alterar os hábitos de vida e de consumo ${ }^{16,17,19}$.

As gestantes percebem que os profissionais da saúde têm dificuldades de compreender a complexidade do problema e desconhecem a abordagem de redução de danos, fator que impacta negativamente nas ações preventivas e no respeito à autonomia da mulher, pois resulta na recusa ou abandono do pré-natal ${ }^{16-19}$.

A redução de danos amplia o cuidado para além da interrupção do uso de drogas. O foco se dá nos impasses que contextualizam o problema como moradia, dificuldade financeira, alimentação, higiene, falta de rede de apoio, gravidez indesejada, segurança pública e assistência social, o que não impede o cuidado à gestante mesmo que a mesma continue fazendo o uso de substâncias. Estas ações estão associadas à melhora da adesão ao pré-natal e dos desfechos da gestação, portanto, a abstinência não pode ser o único objetivo ${ }^{22}$. 
Outras ações que podem ser consideradas redução de danos, mesmo que não nomeadas desta forma pelos estudos, é estimular a reorganização da rotina da gestante e a aproximação com a vivência da maternidade pela prática do autocuidado ${ }^{18}$.

A ausência das gestantes ao pré-natal pode ser atribuída à própria situação social que a droga expõe a mulher ${ }^{17} \mathrm{e}$ nesta perspectiva a atuação do enfermeiro com base na redução de danos, faz-se ainda mais importante. Entretanto, esta proposta de cuidado só foi idenficada em um estudo realizado na Austrália ${ }^{21}$.

A estratégia de encaminhamento foi citada por três dos estudos, em dois aparecem como não qualificados, ou seja, ocorrem simplesmente porque os profissionais da atenção primária não entendem os casos destas gestantes como de sua responsabilidade ${ }^{17,19}$. Existe uma falta de articulação entre a rede de saúde e um modelo de encaminhamento, referência e contra-referência que de fato compartilhe do cuidado das gestantes, não restringindo a complexidade da assistência ao serviço especializado ${ }^{20}$.

Ações sistematizadas e acolhedoras como visita domiciliar, busca ativa, continuidade do acompanhamento individual, apoio a idas em consultas de pré-natal, podem ser realizadas pela equipe multiprofissional na comunidade, mas são brevemente citadas nos estudos ${ }^{16,19,20}$.

O período gestacional constitui um estímulo para as mulheres repensarem o consumo de álcool e outras drogas sobretudo quando estão vinculadas a um cuidado em saúde como o pré-natal ${ }^{19}$, por essa razão, reafirma-se a importância da proximidade do(a) enfermeiro(a) em uma assistência que abranja as dimensões biopsicossociais.

Observa-se que a literatura sobre a temática investigada é escassa e não fornece muitas evidências. Contudo acredita-se que este estudo possa contribuir para o desenvolvimento de novas pesquisas, com delineamento metodológico para avaliação da assistência, produzindo evidências científicas acerca da assistência de enfermagem prestada nos serviços de saúde a gestantes usuárias de crack e cocaína.

\section{Limitações do estudo}

Essa revisão apresenta algumas limitações que precisam ser destacadas: a) a pesquisa foi limitada aos artigos científicos em revistas indexadas, o que resultou na exclusão de trabalhos de conclusão de curso, teses, dissertações, resumos em anais de congresso, relatórios de pesquisa, dentre outros, e b) o restrito número de estudos com metodologia robusta no seu delineamento. Para minimizar a limitação e aumentar a abrangência dos estudos selecionados, utilizou-se três idiomas diferentes e quatro bases de dados.

\section{CONCLUSÃO}

Os estudos apontam para uma lacuna referente às publicações científicas sobre a assistência de enfermagem às gestantes usuárias de crack e cocaína. Evidenciam desafios para contemplar a integralidade e efetividade das ações, principalmente quando a gestante não se sente pronta ou não deseja interromper o consumo de substâncias.

A assistência concentra-se no acolhimento, construção de vínculo e encaminhamentos a serviços especializados, condutas essenciais, porém frequentemente permeadas por estigma e julgamento, o que afasta as gestantes do cuidado em saúde. O enfermeiro tem um papel fundamental no acompanhamento das gestantes nos serviços de saúde o qual precisa ser aprimorado em relação às práticas seguras, éticas e não excludentes, com a implementação de ações diferenciadas com base em estratégias de redução de danos que garantam o direito ao acesso sistematizado a rede de saúde.

Sugere-se a realização de outros estudos com diferentes delineamentos metodológicos por meio de investigações longitudinais e de intervenção, capazes de fornecer consistência e confiabilidade, no intuito de ampliar a clareza e eficácia das ações do enfermeiro e contribuir para a construção do cuidado às gestantes usuárias de crack e cocaína, em diferentes contextos.

\section{REFERÊNCIAS}

1. United Nations Office on Drugs and Crime (UNODC). World Drug Report 2020. New York (NY): United Nations; 2020 [cited 2021 Jun 17]. Available from: https://wdr.unodc.org/wdr2020/field/WDR20_Booklet_2.pdf

2. Bastos FIPM, Vasconcellos MTL, De BoniRB, Reis NB, Coutinho CFS. III Levantamento Nacional sobre o uso de drogas pela população brasileira. Rio de Janeiro: ICICT/FIOCRUZ; 2017 [cited 2020 Jul 13]. 528 p. Available from: https://www.arca.fiocruz.br/handle/icict/34614

3. Fiocruz. Ministério da Saúde (BR). Pesquisa Nacional sobre o uso de crack. Rio de Janeiro: Editora ICICT/FIOCRUZ; 2014 [cited $2020 \mathrm{Jul}$ 15]. Available from: https://www.arca.fiocruz.br/bitstream/icict/10019/2/UsoDeCrack.pdf 
4. Silva FTR, Fernandes CAM, Tamais MLB, Costa AB, Melo SCCS. Prevalence and factors associated with the use of drugs of abuse by pregnant women. Rev. Bras. Saude Mater. Infant. [Internet]. 2020 [cited 2021 Jun 18]; 20(4):1109-15. DOI: https://doi.org/10.1590/1806-93042020000400010

5. Mburu G, Ayon S, Mahinda S, Kaveh K. Determinants of Women's Drug Use During Pregnancy: Perspectives from a Qualitative Study. Matern. Child Health J. [Internet]. 2020 [cited 2021 Jun 14]; 24:1170-8. DOI: https://doi.org/10.1007/s10995-020-02910-w

6. Sebastiani G, Borrás-Novell C, Casanova MA, Pascual TM, Ferrero MS, Gómez RMD, et al. The Effects of Alcohol and Drugs of Abuse on Maternal Nutritional Profile during Pregnancy. Nutrients [Internet]. 2018 [cited 2021 Jun 16]; 10(8):1008. DOI: https://doi.org/10.3390/nu10081008

7. Renner F, Costa B, Figueira F, Ebert J, Nascimento L, Ferrari L, et al. Evaluation of drug use by pregnant women in a teaching hospital in the countryside of Rio Grande do Sul. R. Epidemiol. Control Infec. [Internet]. 2016 [cited 2020 Jun 21]; 6(2):68-73. DOI: http://dx.doi.org/10.17058/reci.v6i2.6976.

8. Errico LSP, Bicalho PG, Oliveira TCFL, Martins EF. The work of nurses in high-risk prenatal care from the perspective of basic human needs. Rev. Bras. Enferm. [Internet]. 2018 [cited 2020 Jul 02]; 71(suppl 3):1335-43. DOI: http://dx.doi.org/10.1590/0034-7167-2017-0328

9. Wronski JL, Pavelski T, Guimarães AN, Zanotelli SS, Schneider JF, Bonilha ALL. Crack use during pregnancy: the experience of women users. Rev. enferm. UFPE on line. 2016 [cited 2020 Jul 05]; 10(4):1231-9. Available from: https://periodicos.ufpe.br/revistas/revistaenfermagem/article/download/11108/12576

10. Chiquetto $\mathrm{CM}$. Puerperas with history of cocaine use and crack: perception of care received during pregnancy and puerperium [monograph]. São Paulo: Universidade de Santo Amaro; 2018 [cited 2020 Jun 4]. Available from: http://docs.bvsalud.org/biblioref/2019/05/995878/tcr-camila-psi-hmec.pdf

11. World Health Organization (WHO). Guidelines for the identification and management of substance use and substance use disorders in pregnancy. Geneva, Switzerland: World Health Organization; 2014 [cited 2020 Jul 11]. Available from: https://www.who.int/publications/i/item/9789241548731

12. Dwyer P.A. Analysis and Synthesis. In: Toronto C., Remington R. (eds) A Step-by-Step Guide to Conducting an Integrative Review. Springer, Cham [Internet]. 2020 [cited 2020 Jul 11]. DOI: https://doi.org/10.1007/978-3-030-37504-1_5

13. Hopia H, Latvala E, Liimatainen L. Reviewing the methodology of an integrative review. Scand. J. Caring Sci. [Internet]. 2016 [cited 2020 Jul 7]; 30:662-9. DOI: https://dx.doi.org/10.1111/scs.12327

14. Palaskar JN. Framing the research question using PICO strategy. J. Dent. Allied Sci. [Internet]. 2017 [cited 2021 Jun 15]; 6(2):55. Available from: https://www.jdas.in/article.asp?issn=22774696; year $=2017$; volume $=6$; issue $=2$; spage $=55$; epage $=55$; aulast $=$ Palaskar

15. Page MJ, McKenzie JE, Bossuyt PM, Boutron I, Hoffmann TC, Mulrow CD, et al. The PRISMA 2020 statement: an updated guideline for reporting systematic reviews. BMJ. [Internet]. 2020 [cited 2021 Jun 17]; 372(71). DOI: https://doi.org/10.1136/bmj.n71

16. Rizzo ER, Messias CM, Valente GSC, Basílio MD, Santos ME, Ferreira SR. The nurse in front of prenatal of a pregnant crack user. Enferm Bras. [Internet]. 2020 [cited 2020 Jul 26]; 19(2):136-47. Available from: https://portalatlanticaeditora.com.br/index.php/enfermagembrasil/article/view/3225/html

17. Lima LPM. Santos AAP, Póvoas FTX, Silva FCL. Nurse's role during prenatal consultation of pregnant drug users. Rev. Espaço Para a Saúde. [Internet]. 2015 [cited 2020 Jul 29];16(3):39-46. Available from: http://espacoparasaude.fpp.edu.br/index.php/espacosaude/article/viewFile/394/382

18. Nicolli T, Gehlen MH, Ilha S, Gabert-Diaz CM, Machado KFC, Nietsche EA. Self-care theory in pregnant women during chemical detoxification from crack: nursing's contributions. Esc. Anna Nery. [Internet]. 2015 [cited 2020 Jul 03]; 19(3):417-23. DOI: https://dx.doi.org/10.5935/1414-8145.20150055

19. Kassada DS, Marcon SS. Waidman, MAP. Perceptions and practices of pregnant women attended in primary care using illicit drugs. Esc. Anna Nery. [Internet]. 2014 [cited 2020 Jun 29]; 18(3):428-34. DOI: https://dx.doi.org/10.5935/1414-8145.20140061

20. Lefevre L, Midmer D, Boyd JA, Ordean A, Graves L, Kahan M, et al. Participant perception of an integrated program for substance abuse in pregnancy. J. ObstetGynecol Neonatal Nurs. [Internet]. 2010 [cited 2020 Jun 29]; 39(1):46-52. DOI: https://dx.doi.org/10.1111/j.1552-6909.2009.01083.x

21. Morris M, Seibold C, Webber R. Drugs and having babies: an exploration of how a specialist clinic meets the needs of chemically dependent pregnant women. Midwifery. [Internet]. 2012 [cited 2020 Jul10]; 28(2):163-72. DOI: https://dx.doi.org/10.1016/j.midw.2011.03.002

22. Serrano Al, Lemos T, Delziovo CR, Rossoni J,Silveira PAF. Abordagem de transtornos por crack e cocaína em gestantes e em bebês: protocolo clínico. Florianópolis (SC): Secretaria da Saúde, 2015 [cited 2020 Jul 10]. Availablefrom: https://www.saude.sc.gov.br/index.php/documentos/atencao-basica/saude-mental/protocolos-da-raps/9216-crack-e-cocainaem-gestantes-e-bebes/file 